\title{
Mercado del cambio de divisas del sector formal versus informal en la ciudad de Estelí en el año 2015
}

\author{
Alberto Cayetano Sevilla Rizo ${ }^{1}$
}

\section{RESUMEN}

El tema sobre mercado del cambio de divisa del sector formal, versus informal en la ciudad de Estelí en el año 2015. Su fin se enmarca en la percepción que tienen los participantes del mercado de divisas de la ciudad de Estelí, tomando en cuenta la caracterización de los clientes, comparar la preferencia, como los factores que intervienen en ambos mercados, esto con el fin de establecer propuestas que vengan a mejorar la utilización de estrategias que conlleven a un mejor servicio al cliente, aprovechando que esta una actividad principal del mercado formal como informal. Es importante destacar que para la recolección de la información, fue necesaria la utilización de encuestas y entrevistas, con el propósito de tener información de primera mano y así brindar recomendaciones que solventen las inquietudes y problemas existentes en ambos mercados. Cabe señalar que el propósito es conocer las preferencia por parte de los clientes y los motivos que lo inducen a elegir al grupo o sector con él que se sienta mayormente identificados, esto es de acuerdo a los criterios que se consideran necesarios y que se dé mejor aprovechamiento en la realización del cambio de divisas por parte de los clientes, además de abordar a los participantes del mercado formal como los participantes del mercado informal, sobre los aspectos más importantes de acuerdo a la percepción y el conocimiento que se tiene sobre las preferencias y algunos inconvenientes que se relaciones con esta actividad. El resultado que se obtuvo, da pautas para hacer las recomendaciones a cada uno de los mercados participantes con el objetivo de comparar y sugerir estrategias que mejoren la realización de esta actividad como es el cambio de divisa.

Palabras clave: Mercado, cambio de divisas, clientes.

Recibido: 25 de febrero de 2016

Aceptado: 13 de junio de 2016

2 Docente del departamento de Ciencias Económicas y Administrativas UNAN-MANAGUA / FAREM-ESTELI Correo electrónico: asevilla_42@yahoo.es 


\title{
Market of currency exchanges of the formal sector vs the informal sector in Estelí in 2015
}

\begin{abstract}
The topic about market of currency exchanges of the formal sector vs the informal sector in Estelí in 2015 is framed from the perception of the participants of the currency market in Estelí. For this purpose, the characterization of the clients, their preference, the factors involved in both markets, were taken into account, with the objective of establishing proposals that lead to a better client service, taking advantage of this principal activity in the formal and informal market. It is important to highlight the need to use surveys and interviews as data collection methods, in order to collect relevant information and give recommendations to solve current problems in both markets. It is relevant to mention the purpose is to know the client's preference and the reasons that make them choose a group or sector in which they find themselves identified, as well as the criteria they consider necessary and that offers better exploitation of in the currency exchange of the clients. The result sets the guidelines to make recommendations to each one of the participant markets, with the objective of comparing and suggesting strategies that improve the realization of the exchange change.
\end{abstract}

Keywords: Market, currency exchange, clients. 


\section{INTRODUCCIÓN}

El mercado del cambio de divisas del sector formal versus informal, se ve implicado en el sistema de cambio el cual está influenciado por la oferta y la demanda de divisas.

El propósito de esta investigación es la percepción que tienen los clientes en el cambio de divisa tanto por el mercado formal como informal, y la determinar los factores que influyen en esto.

Los antecedentes de este estudio se enmarcan en tres investigaciones internacionales, el primer estudio se enfoca en la administración del riesgo en el mercado Forex, un segundo que tiene que ver con el impacto de la compra diaria de dólares del Banco de la República, en la tasa de cambio y un último que trata sobre el análisis comparativo de las metodologías modernas para la valuación de opciones sobre divisas, en general toda esta tratan del comportamiento del dólar, cada una de ella de acuerdo a las necesidades propia de cada uno de los estudios.

El propósito principal del presente estudio en el mercado de cambio de divisas son, el comportamiento y la percepción que tienen los clientes en cada unos de los mercados, esto está vinculado a los factores que intervienen en esta actividad.

Los ejes tienen que ver con los tipo de cambio, los factores que intervienen en la oferta y la demanda de divisas, también se identificaran los tipos de clientes del mercado formal versus mercado informal, las modalidades o tipos de cambio, las modificaciones de la paridad y el control de cambio.

La elaboración de la presente investigación es relevante, ya que permitió conocer cómo funciona, desde un punto de vista más objetivo el mercado cambiario, el cual establece la cotización de una moneda frente a otra, y que depende del control de cambio que exista en el país y de las relaciones entre la oferta y la demanda.

Las variables que se analizaron para dar salida a cada uno de los objetivos son el cliente y el mercado tomándose como indicadores la empresas, persona natural, bancos y cambistas; el segundo sus variables clientes, mercado formal, mercado informal y sus indicadores, tipo de cambio, sistema de tipo de cambio, percepción de los clientes, políticas y como tercero cambio de divisas y sus indicadores estrategias del servicio al cliente, demanda, oferta y tipos de cambio. Además para el análisis de los resultados se tomó en cuenta la ley 732 referente a la oferta y demanda del cambio de divisa en Nicaragua.

\section{MATERIALES Y MÉTODOS}

Según el enfoque mixto es un proceso que recolecta, analiza y vincula datos cuantitativos y cualitativos en un mismo estudio o una serie de investigaciones para responder un problema de investigación (Hernández, Fernández, Baptista, 2006)

Los métodos de investigación mixta representan un conjunto. Es la integración sistemática del método cuantitativo y cualitativo en un solo estudio con el fin de obtener una "fotografía" más completa del fenómeno. Estos pueden ser conjuntados de tal manera que las aproximaciones cuantitativa y cualitativa conserven sus estructuras y procedimientos originales ("forma pura de los métodos mixtos"). Alternativamente, estos métodos pueden ser adaptados, alterados o sintetizados para efectuar la investigación y lidiar con los costos del estudio ("forma modificada de los métodos mixtos"). (Chen, 2006 citado por Sampieri, 2010).

Estos estudios van más allá de la descripción de conceptos o fenómenos, o del establecimiento de relaciones entre conceptos; están dirigidos a responder a las causas de los eventos del mercado 
formal e informal. Como su nombre lo indica, su interés se centra en explicar por qué ocurre un fenómeno y en qué condiciones se da éste, o por qué dos o más variables están relacionadas. Las investigaciones explicativas son más estructuradas que las demás clases de estudios y de hecho implican los propósitos de ellas (exploración, descripción y correlación).

\section{Población y muestra}

El universo de esta investigación lo constituye la población, trabajadores del mercado informal y las empresas de Estelí que realizan las actividades del cambio de divisas.

Los trabajadores del mercado informal conocidos como (cambistas), la población económicamente activa (PEA) comprendida entre 15 y 64 años del municipio de la ciudad de Estelí.

En el primer caso el universo de estudio lo conforman las 17 persona que ejercen el trabajo de cambio de divisas en diferentes lugares de la ciudad de Estelí.

El tipo de muestreo fue probabilístico ya que todos los individuos participantes tuvieron la misma probabilidad de ser seleccionados. Los métodos de muestreo probabilísticos en especial el de estrato son aquellos que se basan en el principio de equisprobabilidad. Es decir, aquellos en los que todos los individuos tienen la misma probabilidad de ser elegidos de acuerdo a cada uno de los segmentos para formar parte de una muestra $\mathrm{y}$, consiguientemente, todas las posibles muestras de tamaño $\mathrm{n}$ tienen la misma probabilidad de ser seleccionadas.

\section{RESULTADO Y DISCUSIÓN}

El estudio de esta investigación trata sobre la percepción que tienen los clientes en el cambio de divisa tanto del mercado formal como informal, y la de determinar los factores que influyen en esto.

\section{Como el primer objetivo es caracterizar los clientes que participan en el cambio de divisas del mercado formal e informal y tomamos los siguientes puntos en consideración.}

Según los resultados que se obtuvieron el sector más frecuentados por los clientes es el sector informal y esto obedece a la rapidez y establecimiento de precio con la que se realiza la transacción. Sin embargo, los que realizan sus transacciones en el mercado formal obedece principalmente a la seguridad.

También es importante señalar que el mercado informal, que está integrado por las personas naturales y que se dedican al cambio de moneda, conocidos por la población como cambistas, se rigen a su vez por la Ley 732, que cita textualmente en su capítulo X (Arto. 489 , que las personas jurídicas que habitualmente se dediquen a la compra y venta de divisas en el territorio nacional deberán llenar los requisitos de inscripción e información que señale el Consejo Directivo del Banco Central. Las personas naturales dedicadas a la misma actividad deberán proporcionar información al Banco cuando así se les requiera.

Se puede conocer que para la clasificación de los clientes del total de encuestado el $69 \%$ afirma que el clasifica a sus clientes de acuerdo a la frecuencia con que realiza sus transacciones un 19

$\%$ de acuerdo al monto y un $12 \%$ otros.

Tomando en cuenta los datos obtenidos, se puede afirmar que los encuestados afirman que unos de los criterios que toman en cuenta para la clasificación de sus clientes es la frecuencia con que realizan dichas transacciones, este es uno de los punto importante dado que la continuidad es lo que les genera mayor ingreso a ambos mercado, y número 2 el monto debido a que esto influye mucho en su clasificación.

Sin embargo, los cambistas clasifican a sus clientes como los empresariales y personales, y según la Ley 
732 caracteriza a clientes como personas naturales y jurídicas (empresas).

En la entrevista realizada a funcionario del Banco del Sistema Financiero Nicaragüense (BSFN), expresó que la categoría utilizada en el mercado formal es cliente mayorista y cliente minorista.

Al preguntar que si cuales son los clientes que generan mayor ingresos, de acuerdo a la percepción que tienen los participantes del mercado informal y según las categorías que expresa según los resultado; indica que es la frecuencia de cambio de divisa como el factor más determinante para su clasificación y, por consiguiente, el que les genera mayor ganancia y esto es proveniente de los clientes personales.

Hay que tomar en cuenta que las veces que realizan cambio de divisa va en dependencia de los hábitos de compras de los clientes, el tiempo con ellos cuentan y las necesidades de acuerdo al giro de sus negocios o necesidades de compra.

\section{Como segundo objetivo se comparo las preferencias de los clientes en el servicio prestado por el mercado formal versus informal, donde se tomaron en cuentas los diferentes criterios tales como:}

Conocer la opinión de los clientes sobre la preferencia para realizar sus cambio más de la mitad de la población encuestada prefieren el mercado informal esto se debe a que la actividad de los cambistas es específicamente solo el cambio de divisa; lo que genera mayor rapidez en las transacciones realizada y este es uno de los punto en estudio y es una de las necesidades principales en los clientes ya que el tiempo es un factor determinante, $\mathrm{y}$ en los otros puntos o lugares que escogen para realizar sus cambios es mucho lento esto se debe a que estas empresas realizan actividades propias del giro de sus negocios lo que permite el mayor porcentaje para este grupo (cambistas).
Por lo tanto los clientes consideran que es muy necesario que existan ambos mercados, tanto el formal como el informal esto obedecen a los criterios como: rapidez, precio, y seguridad, ya que cada uno de los mercados tienen bien marcadas sus diferencias y los clientes siempre están buscando como obtener mayores ingresos, seguridad, entre otros. Esto permite al cliente decida donde realizar su cambio de acuerdo a su necesidad y preferencia según sea su conveniencia.

La motivación que valoran los clientes en elegir unos de los mercados se da, en primer lugar, por el precio lo que indica que los clientes siempre están buscando un poco más de ingreso por sus divisas. En segundo lugar, les interesa la seguridad ya que esto es una actividad muy vulnerable dado que es dinero el que está en riesgo y esto es muy vigilado por los amigo de lo ajeno.

Y por último, la rapidez con que se hacen las transacciones y esto se ve reflejado dado que actualmente el tiempo es un factor determinante en el que hacer de toda la población y también lo expresan en la entrevista realizada a uno de los empresarios de la ciudad de Estelí, dónde manifiesta que los parámetros de mayor importancia que toman en cuenta para preferir unos de los mercado de divisas formal y el mercado informal es el precio, seguridad y la confianza, esto coincide con los resultados obtenidos a los clientes personales.

En la percepción sobre el mercado formal se pudo observar que el $65 \%$ afirma que el mercado formal es seguro, seguido con un $15 \%$ que es por la confianza y en un menor porcentaje en orden de importancia se tiene inseguridad, competencia y respeto.

En este aspecto, se puede referir que la percepción que tienen los clientes del mercado formal es la seguridad, observándose que esto es lo que ofrece mayor confianza para que los clientes utilicen este mercado. 
Y la percepción sobre el mercado informal los clientes afirman que un $47 \%$ de los clientes expresan en primer lugar que el mayor peligro es la inseguridad; en segundo lugar, el $31 \%$ es la confianza, y en un tercer lugar se tiene un $16 \%$ que expresa seguridad y por último, el $6 \%$ respeto.

Este alto porcentaje indica que el mercado informal, debe de tomar medidas en cuanto a los riesgos a que son expuestos tanto los clientes como ellos mismos. Destacando para ambos mercados formal e informal es importante la seguridad, confianza y respeto.

Según la percepción de ,los participantes del mercado informal (cambista) 8 de los encuestados afirman que la razón más importante es la rapidez y 4 de ellos que es el precio y los últimos 4 entrevistados que es la confianza, por lo que se puede decir que la percepción que tienen sobre sus clientes es que lo que tiene mayor peso es la rapidez esta afirmación la hacen por el modelo de trabajo que utilizan y por lo expresado tanto verbal como en las actitudes de sus clientes que quieren ser atendido con rapidez.

El precio y la confianza son clave para este sector y esto se debe a que el cliente siempre está regateando precio buscando mayor ingresos por sus divisas y esto va relacionado con el otro aspecto la confianza ya que el cliente siempre tiene a alguien de su preferencia con quien se siente mayormente identificado.

Con relación a la satisfacción al servicio al cliente Thompson (2006), lo define como es un requisito indispensable para ganarse un lugar en la "mente" de los clientes y por ende, en el mercado meta. Por ello, el objetivo de mantener "satisfecho a cada cliente» ha traspasado las fronteras del departamento de mercadotecnia para constituirse en uno de los principales objetivos de todas las áreas funcionales (producción, finanzas, recursos humanos, etc.) de las empresas exitosas.
Retomando estos conceptos concluyo que la satisfacción al cliente se logra cuando se cumplen con las expectativas en los clientes y en el rendimiento percibido, para que de esa manera, se cumplan los objetivos de ambas partes.

En este aspecto se aplicó la encuesta al 100\% de los cambistas de Estelí, donde el mismo contesto que los clientes se sienten satisfechos con el servicio que se les brinda.

Para el tercer objetivo se van a identificar los factores que intervienen en el cambio de divisas del mercado formal e informal, se llego a los siguientes puntos:

Según la entrevista realizada a los clientes expresan que la existencia del mercado informal ocasiona un equilibrio en el mercado de cambio de divisas y cotejando esta misma pregunta con la que se le realizo a un funcionario de unos de los banco del sistema bancario Nicaragüense, expresó lo siguiente:"es importante ya que mantiene un equilibrio en los precios de compra y venta lo cual evita que exista disparidad o brecha excesiva entre los tipos de cambios". (Funcionario, 2015).

Esta percepción que tiene los clientes sobre lo que ocasiona el mercado informal es como perciben la actividad del cambio de divisa, es decir, los diferentes grupos tienen percepciones bastantes diferenciadas sobre el cambio de divisas posiblemente de acuerdo a sus interese o la simpatía por el uso de uno de los mercados.

Los beneficios que obtiene la población al utilizar el mercado informales que ahorra tiempo y es debido a que es la única actividad que ellos realizan por lo que lo hacen con mayor rapidez y por otra parte obtiene mayores ingresos esto se debe a que los gastos administrativo en este sector es casi nulo lo que le permite jugar con los precios establecido en el sistema bancario de la compra de divisa y vender las divisa más bajo de lo que establece el mercado formal. 
Comparando lo anterior con la entrevista realizada a uno de los empresarios, explica que uno de los beneficios que se tiene y que se valora como parámetro al momento de la elección de cualquiera de los mercados para el cambio de sus divisas es la atención y el precio.

Por lo tanto, según la entrevista realizada a los clientes expresan que la existencia del mercado informal ocasiona un equilibrio en el mercado de cambio de divisas y cotejando esta misma pregunta con la que se le realizo a un funcionario de uno de los bancos de el sistema bancario del país en donde se le pregunto que si consideraban de importancia o no la actividad del mercado informal en la compra y venta de divisa y porque, contestando.

"Es importante ya que mantiene un equilibrio en los precios de compra y venta lo cual evita que exista disparidad o brecha excesiva entre los tipos de cambios". (Funcionario, 2015).

Un grupo menor expresa que ocasiona inestabilidad seguido de un tercer grupo que afirma que ocasiona seguridad esto es de acuerdo a como perciben la actividad del cambio de divisa e la que se puede decir y según el grafico que tienen percepciones bastantes diferenciadas $y$ en un alto porcentaje sobre esta actividad.

Al indagar si utilizan la misma estrategia para la atención a sus clientes según del total de los cambistas encuestado 11 afirma utilizar las mismas estrategias para cada uno de los tipos de clientes y 5 dicen que son diferentes de acuerdo a cada categoría.

Esto muestra claramente que no tiene líneas estratégicas bien definidas a nivel de gremio lo que se ve claramente que cada uno de ellos trabaja de acuerdo a su conveniencia o percibe y se ve claramente que la competencia es un factor fundamental entre ellos mismos.
Cabe mencionar que la estrategia utilizada en la atención al cliente está basada en diferentes puntos donde los participantes del mercado informal valoran como punto claves para determinar la atención que brindaran.

Unos de los punto que más toman en cuenta en primer lugar, es la frecuencia con que los clientes cambian sus divisas, 7 de los 16 encuestado afirman que utilizan estrategia diferente esta actitud para este segmento se refleja que quieren captar a esto tipos de cliente para crear en ello la estrategia de fidelización por que cambian sus divisas con mayor frecuencias.

Uno de los puntos que toman en cuenta es la simpatía y esto se ve claramente por parte de los cliente que tienen sus preferencia ya sea porque los conoce, por recomendación o simplemente se siente que le agrada al hacer sus transacciones con ellos; en tercer punto el monto o la cantidad con que venden, en alguno casos se da que el monto va acompañado con la frecuencia, prácticamente se tendrá que utilizar estrategia diferenciadas para captar a estos tipos de clientes.

En base a las estrategias que utilizan el mercado formal tanto a los clientes individuales y los clientes empresariales; según lo expresado en la entrevista realizada a un funcionario de uno de los banco del sistema financiero del país se le preguntó que si toman medidas para la fidelización de sus clientes y cuáles son esas medidas respondiendo que si, las medidas que toman son Innovación continua, atención especializada, repuesta inmediata a las necesidades del cliente.

Al conocer los factores que intervienen en la oferta y la demanda, en Nicaragua el mercado cambiario está constituido, desde el punto de vista institucional moderno, por: el Banco Central como comprador y vendedor de divisas al por mayor cuando la oferta está total o parcialmente centralizada, la banca comercial como vendedora de divisas al detalle y compradora de 
divisas cuando la oferta es libre o no está enteramente centralizada, las casas de cambio y las bolsas de comercio.

En el mercado de divisas del sector formal e informal en este participan clientes que son las personas jurídicas (empresas) y naturales (persona natural).

Para que se de esta transacción o relación entre ambas partes de la oferta y demanda, la Ley 732 menciona en el Arto. 1, que los participantes, del mercado de cambio de divisas en el territorio nacional sujetos al cumplimiento de las presentes normas serán los siguientes:

Los factores que inciden sobre los mercados de divisas, mercados de creciente volatilidad por las características que hoy los singularizan son: la libertad de movimientos de capital, la rapidez de las transacciones, la existencia de una gama muy amplia de activos financieros, y la importancia de la inversión institucional. El mercado cambiario implica el sistema de cambio el cual está influenciado por la oferta y la demanda de divisas; los factores que intervienen en la oferta y la demanda de divisas, el mercado cambiario, las modalidades o tipos de cambio, las modificaciones de la paridad y el control de cambio. (Morera Cruz, 2004)

La demanda de divisas se origina en las transacciones pasivas o débitos de la balanza: importación de bienes y servicios, pagos por rendimientos de la inversión extranjera en el país, donaciones y remesas enviadas por residentes y exportación de capital no monetario; el componente más estable de la demanda es el referido a la importación de bienes y servicios.

En la entrevista realizada a un funcionario de uno de los bancos se le pregunto que si Considera que los mercados de divisa satisfacen la demanda existente en nuestro país a lo que dijo que sí. También se le pregunto que si como era el procedimiento o las políticas que realizan cuanto no cuentan con la cantidad de divisa que le demandan, su respuesta fue la siguiente. "Existe estrategia de negocio definidas para la oferta y también para cubrir la demanda conforme la tendencia que muestre el mercado"

El origen de la oferta de divisas se origina en las transacciones activas o créditos de la balanza de pagos, tales como: exportación de bienes y servicios, ingresos sobre inversiones del país en el extranjero, donaciones y remesas recibidas por residentes o importación de capital no monetario.

En entrevista realizada se le pregunto que si conocen las políticas que el sistema económico utiliza para ofertar la cantidad de divisa necesaria para mantener la estabilidad económica en el país y de qué manera la podía explicar a lo que argumento lo siguiente.

"Conocemos y cumplimos con las normas tanto nacionales como también observamos las mejores prácticas para la administración del riesgo de mercado, en cuanto al riesgo de mercado emitida por el comité de Basilea". (Funcionario, 2015)

\section{Modalidades del tipo de cambio}

En este punto es importante determinar la variación en el tipo de cambio a lo que unas de las pregunta de la entrevista a funcionario de los banco se les pregunto que si como considera el porcentaje utilizado por el sistema financiero en la relación compra venta su respuesta fue la siguiente.

"Es un porcentaje equilibrado conforme a normas y apetito al riesgo (límite de tolerancia) que la alta gerencia define para este riesgo".

En relación con el margen de utilidad se les pregunto que si consideran que es justo el margen o porcentaje que aplican en la relación compra-venta, afirmando que. "El margen se define por la oferta y la demanda". 


\section{Como cuarto objetivo está la propuesta que se le dio a cada uno de los mercado de acuerdo a los resultado que se obtuvieron es este estudio.}

De acuerdo al estudio realizado y según el análisis de los resultados obtenidos se propone a los participantes del mercado formal y participantes del mercado informal tomar en cuenta los siguientes aspectos para mejorar la participación en el mercado y servicio al cliente:

\section{Mercado formal}

La existencia del mercado formal y el mercado informal es una realidad existente, esto se basa en la opinión de los clientes encuestados que realizan cambio de divisas, y es necesario tomar en cuenta los tres motivos principales para su preferencia como son precio, seguridad y rapidez

Según la percepción que se tiene del mercado formal y la opinión de los encuestados es la seguridad; un motivo que tiene que aprovecharse y utilizarlo a su favor, este punto es de mucha relevancia en este campo debido a que las transacciones que se realizan es en dinero él cliente prefiere siempre desea que sea lo más privado .

De acuerdo a la entrevista realizada a un funcionario de sistema financiero nacional el cambio de divisa ocupa el cuarto puesto en el orden de importancia en las actividades que realizan los bancos y esto viene a ser un elemento de mucho interés para las utilidades que perciben.

Según la encuesta realizada a los clientes un $60 \%$ prefieren el mercado informal y según la opinión de los en los encuestado de los participante del mercado informal (Cambistas) lo que motiva a los clientes es la rapidez o el ahorro del tiempo con el mayor número de ellos.
Por lo que se recomienda a los bancos que utilicen estrategia en donde los clientes que realizan cambio de divisas sean atendidos de manera más personalizada de acuerdo a su clasificación y ser atendido más rápido ya sea:

1. estableciendo cajas que atiendan directamente a este segmento

2. utilizando agente corredores para la atención de clientes exclusivos ya sean en sus negocios o sus empresas.

\section{Mercado Informal}

De acuerdo a la encuesta que se realizó a los clientes se les pregunto que si en cuales de los mercados realiza sus cambios de divisas el $58 \%$ confirmo que lo hace en el mercado informal (cambista) yaa que la preferencia es por el precio seguido por la seguridad y por tercero la rapidez.

En el mercado informal lo que predomina para realizar el cambio de divisa y que motiva a sus cliente para realizar sus transacciones son el precio y la rapidez estos dos aspectos juegan un papel muy importante para la preferencia de sus clientes, Por lo que se le orienta a este sector que mantengan como unas de sus estrategia este punto.

Según la opinión de los clientes sobre los ítems de la percepción del mercado informal es la falta de su seguridad a la hora de la realizar sus transacciones lo que viene a ser un punto en contra para este sector debido a que sus actividades la realizan en lugares muy públicos y sin ninguna vigilancia.

Se propone al mercado informal (cambista) fortalecer esta debilidad que se tiene, esto es según la percepción que tienen los clientes sobre la seguridad, con el objetivo de obtener un mayor posicionamiento en este mercado.

1. Buscar puntos claves de la ciudad de Estelí para poner oficinas de casa de cambio. 
2. Acondicionar estas instalaciones para que reúnan las condiciones necesarias para brindarle mayor seguridad a sus clientes.

3. Para mantener la rapidez se diversificara esta instalación de la siguiente manera:

3.1. A los clientes que realizan sus cambios en sus automóviles diseñar una estructura en la instalación para ser atendido.

3.2. Para los clientes empresariales usar agentes para que lo atiendan en sus negocios o empresas.

3.3. A los clientes personales realizar una infraestructura donde ellos se sientan con comodidad y seguro al realizar sus transacciones.

Esto con el objetico de que los clientes sean atendidos con mayor rapidez y que además se sientan seguro a la hora de realizar sus transacciones.

\section{CONCLUSIONES}

Después de haber analizados los resultados de cada uno de los indicadores de cada una de las variables se puede concluir lo siguiente:

La caracterización de los clientes es un proceso muy importante tanto para el mercado formal e informal; además el método de clasificación de cada uno, ayuda a conocer los criterios que son establecido por ambos mercados para así valorar los parámetros que utilizan de acuerdo a su clasificación esto determinara los ingresos que generaran cada uno de ellos dado por la frecuencia con que realizan sus transacciones.

Al valorar la preferencia por parte de los clientes tanto personales como empresariales de ambos mercado se determinó el nivel de elección, comprobándose que la mayoría prefieren el mercado informal esto por las razones expuesta, las cuales son mayor ingreso y rapidez en el cambio de sus divisas y en un porcentaje menor para el mercado formal es por la mayor seguridad que este tiene.
Los motivos principales al preferir unos de los mercado el principal es el precio y esto lo expresaron tanto el cliente personal como empresarial y es que siempre están esperando mayor ingresos por sus divisas y en este aspecto prefieren el mercado informal por que afirman que el que tiene un mejor precio.

La seguridad es el otro factor importante y el que le presente una mejor alternativa, con mejores condiciones para transacciones es el mercado formal.

Dentro de la preferencia y que va con el mercado informal es la rapidez y es uno de los factores fundamentales que tiene que explotar, ya que es el segundo punto de importancia en el cambio de divisas, esto es dado por que este grupo es la única actividad que realiza y en relación con el mercado informal tienen que hacer actividades de acuerdo al giro de sus negocios.

Los factores que interviene en el cambio de divisas son las estrategias de atención al cliente que tiene cada uno de los mercados y cada uno de ellos lo tienen bien segmentado y de manera similar el mercado informal los tiene como empresariales y personales y el mercado informal como mayorista y minorita y cada uno de los mercados el objetivo principal es la fidelización de los clientes de acuerdo a los puntos establecidos anteriormente.

La oferta y la demanda permite tanto al mercado formal como informal establecer el precio de compra como de venta ya que es el movimiento que tiene el mercado y las necesidades la que determinara el precio tanto de compra como de venta pero siempre dentro de los parámetros establecido por el banco central de Nicaragua

Por lo tanto, se comprobó que tanto al mercado formal como al mercado informal, deben prestar un mejor servicio a sus clientes y a si tener una competencia más justa en ambos mercado porque de acuerdo a la 
encueta realizada a los cliente y la entrevista a uno de los funcionario del sistema bancario de Nicaragua la existencia del mercado informal viene a equilibrar al mercado de divisa.

Para finalizar, el cambio de divisa tanto para el mercado formal como informal se ve determinado por la caracterización de los clientes y la preferencia que tienen cada uno de estos segmentos en que están divididos y estos es de acuerdo a los diferentes criterios establecidos. Tomando en cuenta como unos de los factores principales la oferta y la demando de divisas que existen en cada uno de los mercados y el mecanismo o proceso que tiene este en su regulación.

\section{BIBLIOGRAFIA DE REFERENCIA}

Abraham Silbershatz, H. F. (2002). Fundamento de base de datos. Madrid : Concepcion Fernandez Madrid.

Avendaño, N. (20 de octubre de 2010). Recuperado el 20 de agosto de 2014, de NestorAbendaña http://nestoravendano.wordpress.com/la-politicacambiaria-de-nicaragua-en-2010/

BCN. (2010). bcn. el 20 de agosto de 2014, de billetes_monedas: htp://www,bcn.gob. ni/billetes_monedas/resena.php

BCN. (2010). bcn. Recuperado el 20 de agosto de 2014, de estadisticas: http://www. bcn.gob.ni/estadisticas/mercados_cambiarios/ index.php

BCN. (2010). www.bcn.gob.n. Recuperado el 20 de agosto de 2014, de estadisticas: http://www.bcn.gob.ni/estadisticas/mercados cambiarios/index.php

Brasil, P. a. (2000). CRM Series Marketing 1 TO1. Brasil: Peper and Roger Group de Brasil. Casanueva Rocha, C. (2000). Organización y gestión de empresas turisticas. Madrid: Piramides. CEPAL. (2012). Poblacion, territorrio y desarrollo sostenible. Ecuador: Naciones unidas.

Enciclopedia Coorporativa;. (s.f.). Enciclopedia
Finanaciera. Recuperado el 08 de Marzo de 2015, de Participante en los mercados de divisas: http:// www.enciclopediafinanciera.com/mercadosfinancieros/divisas/participantes-en-los- mercadosde-divisas.htm

Funcionario. (23 de 12 de 2015). Mercado de cambio de divisas. (A. Sevilla, Entrevistador)

FXto. (27 de enero de 2014). www.efxto.com/articulosforex. Recuperado el 26 de agosto de 2014, de 2896-los-factores-que-afectan-a-los-tipos-decambio-entre-divisas: https://www.efxto.com/ articulos-forex/2896-los-factores-que-afectan-alos-tipos-de-cambio- entre-divisas

Gastélun, R. G. (2007). Comunicacion y cultura organizacional en empresas chinas y japonesas. Recuperado el 28 de diciembre de 2015, de www.eumed.net: http://www.eumed.net/librosgratis/2007a/221/1t.htm

Gómez, G. R. (1996). Introducción a la Investigación cualitativa. En G. R. Gómez, Metodología de la Investigación cualitativa (pág. 32). Granada España: Aljibe.

Gonzalez, M. E. (2002). Conceto de mercado y sus tipos. Recuperado el 08 de Marzo de 2015, de http:/www.gestiopolis.com/canales/economia/ articulos/42/conmercadhel.htm

Kather, P. Direccion de Markething. Shoping House. Kotler, P. (2007). Direccion de marketing. Shoping House.

León, C., \& Miranda, M. (2003). Análisis macroeconómico. Lima: Universidad Católica Santos Toribios de Mongrovejo.

Mendez A., C. (2009). Metodología, diseño y desarrollo del proceso de investigación con enfasis en las ciencias empresariales . Mexico: LIMUSA.

Morera Cruz, O. J. (enero de 2004). Fundamentos de economia. Recuperado el 20 de agosto de 2014, de http://www.gestiopolis.com/canales/economia/ articulos/70/tipcammor.htm

Tamayo, T. M. (1994). El proceso de la investigación científica. Mexico: LIMUSA-Noriega 


\section{Editores}

wordpress. (2014). Definicion de cliente.

Recuperado el 16 de enero de 2015, de http://definicion.de/cliente/\#ixzz3P2h7SvCE www.economia48.com. (s.f.). Recuperado el 25 de diciembre de 2014, de cambista/ cambista.htm: http://www.economia48.com/spa/d/ cambista/cambista.htm 